\title{
Cosa deve sapere ogni pediatra sulla comunicazione aumentativa alternativa (CAA)
}

\author{
CYNTHIA LA MANNA', ISABELLA PRINA', MICHELE CAVALLERI', PATRIZIA CONTI', ANGELO SELICORNI² \\ 'UO di Neuropsichiatria Infantile e dell'Adolescenza, ${ }^{2}$ UOC di Pediatria, ASST Lariana, Como
}

$\grave{E}$ un approccio dai vari volti, ma con lo scopo univoco di offrire alle persone con bisogni comunicativi complessi la possibilità di utilizzare canali che si affiancano alla comunicazione orale. La comunicazione aumentativa alternativa (CAA) offre numerose potenzialità, ma è ancora poco diffusa nel nostro Paese. "Comunicare è un diritto di tutti, favorirne la possibilità è un dovere sociale".

\section{CHE COS'È LA CAA?}

Si tratta di un'area della pratica clinica che cerca di compensare la disabilità temporanea o permanente di soggetti con bisogni comunicativi complessi. È una Comunicazione interattiva e viene usata da entrambi i partner comunicativi: quello verbale e quello con deficit di linguaggio o non verbale: quando viene utilizzata dal partner comunicativo verbale è a supporto della comprensione del linguaggio parlato (recettiva); spesso si avvale di gesti o segni e immagini e utilizza un doppio input comunicativo sia visivo che uditivo.

È Aumentativa perché ha lo scopo principale di potenziare, ampliare ed espandere (in inglese augmentative $^{1}{ }^{1}$ la comunicazione e il linguaggio supportando tutte le potenzialità comunicative della persona a partire dalle vocalizzazioni e dal linguaggio verbale esistente.

È Alternativa perché utilizza modalità alternative alla comunicazione tradizionale: si avvale di gesti, segni, ausili per la comunicazione e tecnologia avanzata e rientra nella tecnologia assistiva.

\section{CHE COSA NON È...}

Non è una tecnica riabilitativa perché non può essere patrimonio solo

\section{WHAT PAEDIATRICIANS NEED TO KNOW ON AUGMENTATIVE ALTERNATIVE COMMUNICATION (AAC)}

(Medico e Bambino 2020;39:513-517)

\section{Key words}

Augmentative Alternative Communication (AAC), Double communicative input, Complex disabilities, Assistive technology,

\section{Summary}

Augmentative Alternative Communication (AAC) is an area of clinical practice that tries to compensate for the temporary or permanent disability of individuals with complex communication needs. It uses gestures or signs and images and utilises a double communicative input, both visual and auditory. It is Augmentative because its main purpose is to enhance and expand ("augmentative" 1983 ISAAC) communication and language, supporting all the communicative potential of the person. It is Alternative because it uses an alternative method to traditional communication: it utilises gestures, signs, aids for communication and advanced technology, falling within Assistive Technology (AT). AAC users are mainly those with complex (cognitive and communicative) disabilities. Moreover, it is also used in all those temporary situations in which communication is hindered by traumatic factors (intensive care, first aid) or linked to the issue of integration / inclusion. For example, foreign people can benefit from the use of AAC in order to reduce discomfort in social relations and language learning. The Authors highlight the potential of AAC both on the basis of scientific and clinical evidence and describe the evolution of two clinical cases followed at the Child and Adolescent Neuropsychiatry Unit in Como (Italy).

del terapista, ma deve essere adottata e condivisa da tutti i contesti di vita del suo fruitore, in ogni momento e luogo della sua vita: risponde così alle continue esigenze di cambiamento ed è quindi un sistema flessibile, su misura per ogni persona.

Il modello di intervento risulta essere esclusivamente e imprescindibilmente partecipativo e prevede l'attivazione di tutto il contesto di vita del soggetto interessato cioè la famiglia, la scuola, i Servizi sociali, le strutture sanitarie, i Centri ludico-sportivi-ricreativi, la struttura religiosa e la biblioteca che frequenta. Tale attivazione è subordinata a un percorso formativo presso Centri dedicati.

\section{CHI SONO GLI UTENTI?}

Principalmente quei soggetti in età evolutiva e in età adulta, portatori di una disabilità complessa (cognitiva e di comunicazione) capaci di utilizzare 
strategie per esprimere bisogni comunicativi legati a scelte, emozioni e stati d'animo.

Inoltre tutte quelle situazioni temporanee in cui la comunicazione è ostacolata per impedimenti legati a fattori traumatici (Terapia Intensiva, Pronto Soccorso) oppure legate alla problematica di integrazione/inclusione: le persone straniere possono beneficiare dell'utilizzo della CAA al fine di ridurre il disagio nella comunicazione interpersonale, nelle relazioni sociali e nell'apprendimento della lingua.

\section{QUANDO UTILIZZARE LA CAA?}

È importante considerare che l'unico prerequisito fondamentale per la presa in carico con la CAA è l'attivazione del contesto in termini di collaborazione continuativa nel tempo e partecipazione al percorso formativo sia del soggetto che della collettività, mentre età, livello cognitivo o gravità di compromissione non sono fattori così rilevanti.

\section{PERCHÉ UTILIZZARE LA CAA?}

La CAA migliora il linguaggio verbale implementando le abilità di comunicazione e interazione ${ }^{2}$. Soggetti con disturbo dello spettro autistico migliorano il loro uso del linguaggio parlato comprensibile se esposti a simboli grafici, visivi e parole durante l'interazione con il partner comunicativo, in situazioni quotidiane ${ }^{3}$.

La CAA con tavole di comunicazione mediante immagini ha aumentato la frequenza e la lunghezza delle espressioni verbali durante la lettura di storie ${ }^{4}$ e ha implementato sia l'attenzione congiunta che le capacità grafico-rappresentative, come emerso da ricerche in ambito clinico.

\section{QUALI I PRESUPPOSTI TEORICI DELLA CAA?}

I capisaldi teorici alla base del modello di intervento con la CAA sono due: i neuroni specchio e l'ICF (classificazione internazionale del funziona- mento, delle disabilità e della salute, redatta dall'Organizzazione Mondiale della Sanità).

I primi sono un sistema automatico che entra in risonanza con le azioni effettuate da un altro, attivando lo stesso partner motorio necessario a compiere le azioni osservate, senza agire però concretamente.

La teoria dei neuroni specchio mette in evidenza come lo snodo chiave che differenzia l'uomo dai primati sul piano comunicativo non è stato tanto il linguaggio, quanto la funzione comunicativa e sociale attraverso gesti naturali e specifici quali indicare e mimare ${ }^{5,6}$. Dal momento che la capacità umana di comunicare si è sviluppata prima dai gesti e poi dal linguaggio è un sistema attivo fin dalla nascita: infatti i neonati nascono con una serie di competenze innate che favoriscono lo sviluppo della comunicazione, ma tali competenze necessitano dell'interazione con l'altro per svilupparsi e implementarsi ${ }^{7,8}$. Ecco che ogni atto comunicativo presuppone almeno due partner che attraverso la ripetizione regolare di sequenze creano un terreno comune noto e condiviso che permette la comunicazione intenzionale in una turnazione di gesti e parole. Questa capacità dell'uomo di collaborare con altri uomini verso fini congiunti e di agire con una intenzionalità comunicativa condivisa è unica e specifica della comunicazione umana ${ }^{9,10}$ e rende dunque fondamentale l'intervento di CAA nei soggetti con bisogni comunicativi complessi.

L'ICF, redatta dall'Organizzazione Mondiale della Sanità, ha portato a un grande cambiamento dello sguardo sulla disabilità, che non viene più classificata in base alla conseguenza della malattia, bensì in base alle componenti della salute che sono intese come fattori fondamentali di salute e benessere ${ }^{11}$. Se guardiamo dunque alla disabilità come al risultato dell'interazione fra fattori individuali e fattori contestuali/ambientali ${ }^{12}$, anche la comunicazione risulta tra questi e nel caso di un disturbo complesso o situazioni contingenti/ambientali problematiche può essere causa di un ampliamento della disabilità così come in presenza di strategie e facilitatori può risultare un fattore assolutamente rilevante per una riduzione della stessa. L'intervento di CAA ha lo scopo di creare strumenti utili a promuovere e sviluppare la comunicazione spontanea con conseguente riduzione della disabilità (ICF), incrementando la partecipazione attiva e l'intenzionalità comunicativa nei diversi ambienti.

\section{QUALI GLI STRUMENTI?}

Gli strumenti e le tecniche utilizzate nella CAA sono diversi e molteplici in base alla patologia, all'età ai bisogni personali e alle caratteristiche cliniche. Una prima distinzione si basa sul tipo di sistema utilizzato: corporeo, cioè segni manuali, gesti e vocalizzazioni, CAA non assistita, oppure extracorporeo, cioè disegni, fotografie, simboli tridimensionali o lineari, CAA assistita. Quando il sistema assistito si avvale del supporto di dispositivi tecnologici o ausili informatici, in base al livello di tecnologia si distinguono sistemi di CAA a bassa tecnologia oppure ad alta tecnologia ${ }^{13}$. Il linguaggio dei segni è un esempio di CAA non assistita, i PECS (sistema di comunicazione per scambio di simboli), le tavole comunicative, gli Inbook (libri con il testo scritto in simboli) sono esempi di CAA assistita.

\section{CAA E STRUTTURE SANITARIE: L'ESPERIENZA DI COMO}

L'ambulatorio di Neuropsichiatria infantile si occupa di diagnosi, cura, riabilitazione e presa in carico dei minori che soffrono di malattie neurologiche, psichiatriche, disturbi neuropsicologici e disabilità di vario grado. L’intervento è allargato alla famiglia e all'ambiente di vita e viene effettuato da una équipe multidisciplinare.

Tra i vari interventi, quello della CAA sui minori con difficoltà di comunicazione linguistica occupa un posto di rilievo e viene proposto sia in ambito ambulatoriale che in via sperimen- 


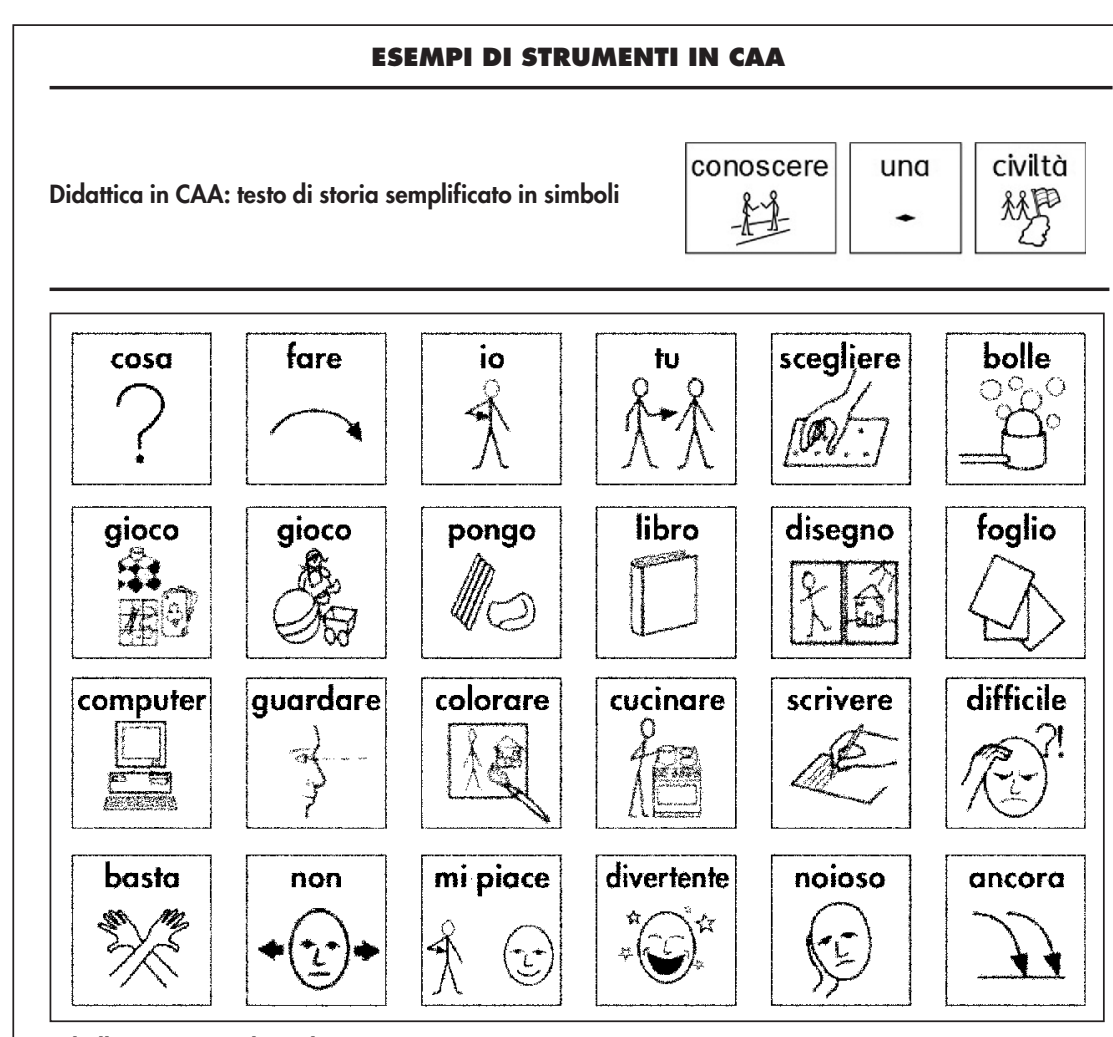

Tabella a tema per la scelta

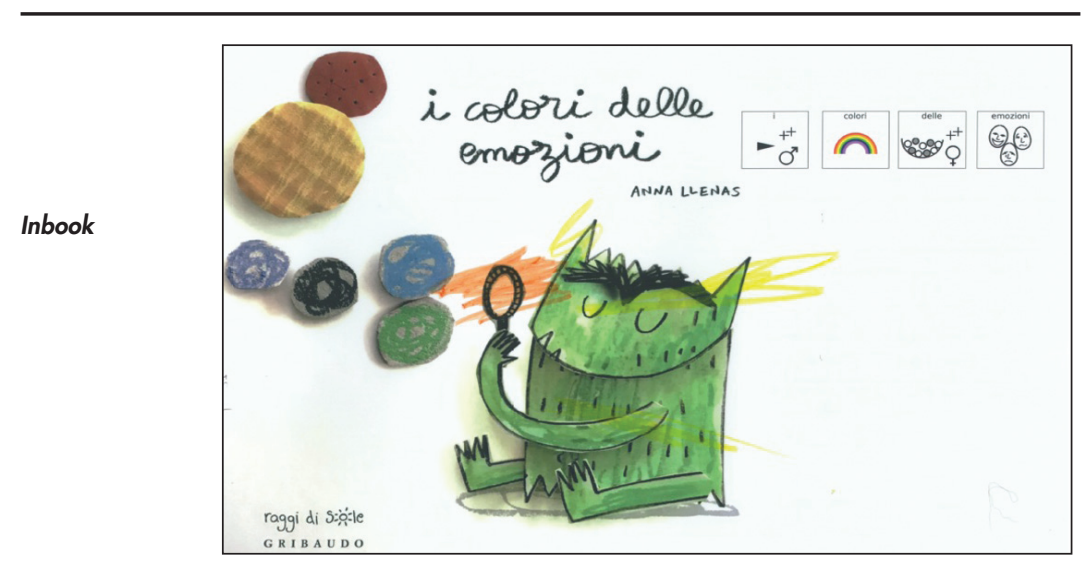

Libri modificati su misura

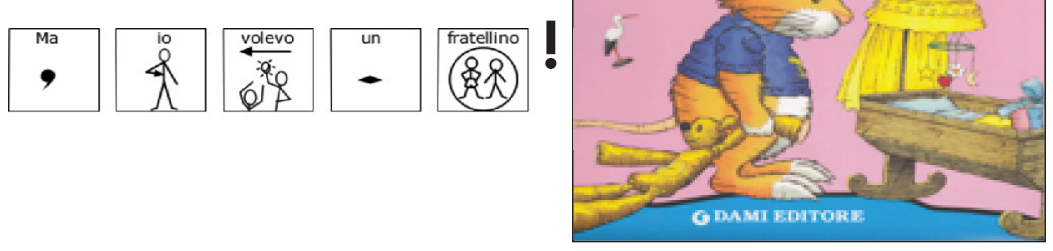

Per saperne di più: www.sovrazonalecaa.org - www.csinbook.altervista.org - www. isaacitaly. it tale presso il reparto di Pediatria dell'ospedale Sant'Anna di Como, dove l'équipe dedicata alla CAA fornisce una consulenza al reparto di Genetica pediatrica, diretta dal dottor Angelo Selicorni, finalizzata ad attivare percorsi di CAA, fornendo materiali informativi e supervisioni ai Centri riabilitativi di riferimento.

L'obiettivo di incontrare genitori e bambini affetti da sindromi genetiche, perlopiù rare, è di individuare gli strumenti più efficaci per sviluppare l'intenzionalità comunicativa e la capacità di attenzione condivisa all'ascolto così da sostenere la comunicazione (comprensione e linguaggio), creando situazioni/ambienti facilitanti e motivanti. Uno di questi strumenti è il libro illustrato in simboli, personalizzato, cioè fatto sull'esperienza e il vissuto del bambino, oppure il libro in simboli costruito "su misura" per il bambino, quindi modificato dal testo originale $^{11}$. Per offrire miglior accoglienza ai pazienti e alle loro famiglie sono stati "etichettati" con simboli di CAA gli ambienti sia del reparto di Pediatria che del Pronto Soccorso pediatrico e all'interno della libreria presente in reparto, è stata allestita una piccola sezione di libri in simboli CAA.

I libri in simboli sono un valido strumento anche quando traducono fedelmente il testo originale (Inbook) e vengono usati nella nostra Unità Operativa di Neuropsichiatria Infantile e dell'Adolescenza (UONPIA) nei laboratori con piccoli utenti con bisogni comunicativi complessi, tra cui anche stranieri esposti a bi-trilinguismo, così da condividere un modello di lettura ad alta voce anche con i genitori che spesso necessitano di percorsi che supportino il legame affettivo con i figli e la capacità empatica. I laboratori vengono anche svolti nelle scuole sia dell'infanzia che primaria e secondaria di primo grado così da generalizzare la proposta di lettura ad alta voce di libri in simboli, favorendo l'integrazione e l'inclusione del soggetto con bisogni comunicativi complessi nella sua classe e nell'ambiente scolastico.

La CAA e gli strumenti di CAA, utilizzati nel progetto di intervento ambulatoriale sia durante i laboratori 
di lettura sia nei trattamenti neuropsicomotori individuali e di gruppo, hanno permesso a molti dei nostri utenti e alle loro famiglie di migliorare le relazioni sociali, aumentando le possibilità di integrazione, inclusione nei diversi contesti di vita, potenziando il coinvolgimento dei partner comunicativi sia genitori che insegnanti e coetanei. Di seguito due casi clinici che illustrano le modalità di intervento con la CAA.

\section{Caso 1: sindrome da alterazione globale dello sviluppo psicologico non specificata}

Il bambino nasce nel 2005. All'età di tre anni viene fatta una valutazione neuro-psicomotoria che evidenzia un difetto di regolazione del comporta- mento, con instabilità psicomotoria, associato a un quadro di disfasia. Presenta un potenziale cognitivo, ma essendo esposto a tre lingue (filippino, inglese, italiano) la comprensione è deficitaria così come l'espressione linguistica è gravemente compromessa. In seguito alla valutazione inizia il trattamento psicomotorio presso la UONPIA di Como con frequenza bisettimanale. All'età di sei anni l'équipe multidisciplinare decide di inserire il bambino all'interno del Progetto regionale "Supporto delle gravi disabilità della comunicazione in età evolutiva" che, con la formazione e supervisione dell'équipe del Centro sovrazonale di Comunicazione Aumentativa, diretta dalla dottoressa Costantino, si occupa di formare in CAA le UONPIA della

\section{CASO 1: CAMBIAMENTI SIGNIFICATIVI AVVENUTI CON LA CAA}

\begin{tabular}{l|l}
\hline Prima dell'intervento in CAA & Dopo l'intervento in CAA \\
\hline $\begin{array}{l}\text { Bambino silente, agisce i bisogni } \\
\text { e usa poco la comunicazione non verbale }\end{array}$ & $\begin{array}{l}\text { Ecolalia differita contestuale e imitazione } \\
\text { gestuale del simbolo associato alla parola } \\
\text { (basta/ancora) } \\
\text { Olofrase contestuale al bisogno }\end{array}$ \\
\hline Scrittura guidata e imitativa al PC & $\begin{array}{l}\text { Miglioramento della comprensione } \\
\text { della lingua italiana e produzione di parole } \\
\text { fino alla produzione di semplici frasi legate al } \\
\text { contesto }\end{array}$ \\
\hline Isolamento con i pari & $\begin{array}{l}\text { Scrittura spontanea di singole parole al PC } \\
\text { con iniziale significato contestuale. Lettura di } \\
\text { simboli, ma anche di parole ad alta } \\
\text { familiarità senza simbolo associato }\end{array}$ \\
\hline $\begin{array}{l}\text { Iniziali rapporti con i coetanei e maggior } \\
\text { coinvolgimento nel piccolo gruppo classe fino } \\
\text { alla partecipazione a momenti extrascolastici } \\
\text { come compleanni e pizze di gruppo }\end{array}$
\end{tabular}

Tabella I

\section{CASO 2: CAMBIAMENTI SIGNIFICATIVI AVVENUTI CON LA CAA}

\begin{tabular}{l|l}
\hline Prima dell'intervento in CAA & Dopo l'intervento in CAA \\
\hline $\begin{array}{l}\text { Bambino con assenza di produzione } \\
\text { verbale in lingua italiana/poche parole } \\
\text { in turco. Vocalizzi afinalistici }\end{array}$ & $\begin{array}{l}\text { Ripetizione di parole bisillabiche e } \\
\text { trisillabiche con supporto del simbolo, } \\
\text { aumento delle parole nella lingua madre, } \\
\text { riduzione importante dei vocalizzi afinalistici }\end{array}$ \\
\hline $\begin{array}{l}\text { Scarsa comprensione del messaggio } \\
\text { verbale sia in lingua turca che in italiano }\end{array}$ & $\begin{array}{l}\text { Miglioramento della comprensione della } \\
\text { lingua italiana e produzione spontanea di } \\
\text { parole legate alla presentazione del simbolo }\end{array}$ \\
\hline $\begin{array}{l}\text { Atteggiamento ipo-reattivo di fronte } \\
\text { alle proposte }\end{array}$ & $\begin{array}{l}\text { Partecipazione alle attività strutturate } \\
\text { e semi-strutturate }\end{array}$
\end{tabular}

Tabella II

Lombardia e i contesti di vita dei loro utenti. Dopo una valutazione sulle capacità comunicative emerge che il bambino ha un disturbo di comprensione significativo e fatica a comprendere le parole se isolate dal contesto: si comporta come una persona straniera alle prese con una lingua sconosciuta; il suo livello cognitivo è migliore del suo livello linguistico.

Le proposte di intervento messe in atto durante l'approccio neuro-psicomotorio integrato alla CAA hanno comportato un utilizzo massiccio di libri su misura, l'utilizzo degli strumenti di CAA in simboli WLS (Widgit Literacy Symbols) e l'utilizzo in un primo momento di un ausilio a uscita in voce (32 caselle) e successivamente di un tablet dotato di un software per griglie con simboli in CAA (Figura 1). Con la crescita del bambino si è considerata la necessità di lavorare sulle capacità/autonomie sociali ed è stato proposto un percorso condiviso con un altro utente con bisogni comunicativi complessi, utilizzando la CAA come linguaggio comune a entrambi gli utenti. Durante la presa in carico con la CAA sono stati anche proposti laboratori di lettura ad alta voce con libri in simboli Inbook sia presso il Servizio di Neuropsichiatria sia presso la scuola primaria e secondaria di primo grado che tutt'oggi frequenta con una buona riuscita sia dal punto di vista degli apprendimenti (la CAA lo supporta nella comprensione dei testi e nelle verifiche personalizzate) sia dell'integrazione e inclusione all'interno della classe.

La Tabella I evidenzia i cambiamenti significativi avvenuti.

\section{Caso 2: disturbo generalizzato dello sviluppo non ancora specificato (ICD 10, F 84.9)}

Il bambino, originario della Turchia, nasce a maggio 2016 a Como, con parto eutocico dopo una gravidanza fisiologica. Riscontro di ipoglicemia in prima giornata di vita trattata con infusione con soluzione glucosata. Il bambino ha raggiunto la deambulazione autonoma ai 13 mesi. All'età di tre anni la valutazione neuro-psicomotoria ha evidenziato un comporta- 


\section{MESSAGGI CHIAVE}

$\square$ Comunicare è un diritto di tutti, favorire la possibilità di comunicare è un dovere sociale.

$\square$ La CAA rappresenta più di una pratica clinica dal momento che costituisce una vera e propria lingua per chi è portatore di bisogni comunicativi complessi. $\square \grave{E}$ di supporto alla relazione, allo scambio comunicativo fra pari, sostiene le autonomie sociali e gli apprendimenti in ogni contesto di vita.

mento ipoattivo, assenza di gioco strutturato, comportamenti e azioni non finalizzati e stereotipati, grave ritardo nello sviluppo del linguaggio. In ambiente domestico è esposto prevalentemente alla lingua di origine, il turco. I genitori hanno riferito che il bambino dice solo poche parole nella sua lingua ("mamma", "finito", "ancora”) e che tende a giocare da solo, senza ricercare l'interazione con gli altri; le autonomie personali presentano un marcato ritardo. Inizia così un trattamento individuale psicomotorio con cadenza bisettimanale integrato all'uso della CAA dal momento che la comprensione, l'espressione linguistica e l'intenzionalità comunicativa sono gravemente compromesse e limitate a vocalizzi spesso afinalistici.

Le proposte di intervento messe in atto durante l'intervento neuro-psicomotorio hanno integrato l'uso della CAA per sostenere la comprensione del messaggio verbale e lo scambio comunicativo. Sono state proposte at- tività ludiche, partendo da un livello senso-motorio, supportate dai simboli di CAA (Figura 1) che la terapista della neuro- e psico-motricità dell'età evolutiva ha usato in entrata per supportare la comprensione dell'azione e stimolare l'attenzione condivisa. Il rapido cambiamento che si è evidenziato dopo circa due mesi di intervento ha permesso la proposta di lettura di semplici libri in simboli CAA e l'ampliamento del setting con proposte di attività ludiche strutturate e semistrutturate.

La Tabella II evidenzia i cambiamenti significativi.

\section{Indirizzo per corrispondenza:}

Angelo Selicorni

e-mail: angelo.selicorni61@gmail.com

\section{Bibliografia}

1. ISAAC Italy. Società Italiana per la Comunicazione Aumentativa Alternativa, sezione italiana. http://www.isaacitaly.it/caa.htm. Ultimo accesso 12 ottobre 2019.
2. Millar D, Light J. Eshosser R. The impact of AAC on natural speech development: a meta analysis. Atti della 9a conferenza biennale dell'ISAAC. Washington (DC), 2000, pagg. 740-1.

3. Romsky MA, Sevick RA. Breaking the speech barrier: language developmental through augmented means. Baltimore: Paul $\mathrm{H}$. Brooks, 1996.

4. Dexter ME. The effects oh aided language stimulation upon verbal output and augmentative communication during storybook reading for children with pervasive developmental disabilities. Johns Hopkins University, 1998, 492 pagg.

5. Costantino MA, Anastasi S, Bergamaschi $\mathrm{E}$, et al. Costruire libri e storie con la CAA. Gli IN-book per l'intervento precoce e l'inclusione. Erikson, 2011, pagg. 43.

6. Rizzolati G, Sinigallia C. So quel che fai: il cervello che agisce e i neuroni a specchio. Milano: Raffaello Cortina editore, 2006.

7. Costantino MA, Anastasi S, Bergamaschi $\mathrm{E}$, et al. Costruire libri e storie con la CAA. Gli IN-book per l'intervento precoce e l'inclusione. Erikson, 2011, pag. 45.

8. Gergely G, Watson JS. The social biofeedback model of parrental affect mirroring: the developmental of emotional self-awareness and self control in infancy. Int J Psychoanal 1996;77(Pt 6):1181-212.

9. Costantino MA, Anastasi S, Bergamaschi $\mathrm{E}$, et al. Costruire libri e storie con la CAA. Gli IN-book per l'intervento precoce e l'inclusione. Erikson, 2011, pagg. 47-8.

10. Tommasello M. Le origini della comunicazione umana. Milano: Raffaello Cortina editore, 2009.

11. National Joint Committee for the Communication Needs of Persons With Severe Disabilities. Guidelines for meeting the communication needs of persons with severe disabilities. 1992. https://www.asha.org/policy/GL1992-00201.

12. Buono S, Zagaria T. ICF - classificazione internazionale del funzionamento delle disabilità e della salute. Ciclo evolutivo e disabilità. 2003.

13. Costantino MA, Anastasi S, Bergamaschi $\mathrm{E}$, et al. Costruire libri e storie con la CAA. Gli IN-book per l'intervento precoce e l'inclusione. Erikson, 2011, pag. 55 e pagg. 79-135. 